\title{
New clinical research strategies in thoracic oncology: clinical trial design, adaptive, basket and umbrella trials, new end-points and new evaluations of response
}

\author{
Jessica Menis ${ }^{1}$, Baktiar Hasan² and Benjamin Besse ${ }^{3}$ \\ Number 6 in the Series "Topics in Thoracic Oncology" \\ Edited by G. Zalcman and N. Girard
}

Affiliations: 'Medical Dept, European Organisation for Research and Treatment of Cancer (EORTC), Brussels,
Belgium. ${ }^{2}$ Statistical Dept, European Organisation for Research and Treatment of Cancer (EORTC), Brussels,
Belgium. ${ }^{3}$ Dept of Cancer Medicine/Thoracic Unit, Gustave Roussy Cancer Campus Grand Paris, Villejuif, France.

Correspondence: Benjamin Besse, Dept of Cancer Medicine/Thoracic Unit, Gustave Roussy Cancer Campus, 117 rue E.Vaillant, 95805 Villejuif, France. E-mail: benjamin.bessedgustaveroussy.fr

ABSTRACT In the genomics era, our main goal should be to identify large and meaningful differences in small, molecularly selected groups of patients. Classical phase I, II and III models for drug development require large resources, limiting the number of experimental agents that can be tested and making the evaluation of targeted agents inefficient.

There is an urgent need to streamline the development of new compounds, with the aim of identifying "trials designed to learn", which could lead to subsequent "trials designed to conclude".

Basket trials are often viewed as parallel phase II trials within the same entity, designed on the basis of a common denominator, which can be a molecular alteration(s). Most basket trials are histology-independent and aberration-specific clinical trials. Umbrella trials are built on a centrally performed molecular portrait and molecularly selected cohorts with matched drugs, and can include patients' randomisation and strategy validation. Beyond new designs, new end-points and new evaluation techniques are also warranted to finally achieve methodology and clinical improvements, in particular within immunotherapy trials.

@ERSpublications

New clinical research strategies are needed to bring "trials designed to conclude" into thoracic oncology http://ow.ly/yKfWz

\begin{abstract}
Previous articles in this series: No. 1: Girard N. Thymic epithelial tumours: from basic principles to individualised treatment strategies. Eur Respir Rev 2013; 22: 75-87. No. 2: Dooms C, Muylle I, Yserbyt J, et al. Endobronchial ultrasound in the management of nonsmall cell lung cancer. Eur Respir Rev 2013; 22: 169-177. No. 3: Lang-Lazdunski L. Surgery for nonsmall cell lung cancer. Eur Respir Rev 2013; 22: 382-404. No. 4: Bergot E, Levallet G, Campbell K, et al. Predictive biomarkers in patients with resected non-small cell lung cancer treated with perioperative chemotherapy. Eur Respir Rev 2013; 22: 565-576. No. 5: Cortot AB, Jänne PA. Molecular mechanisms of resistance in epidermal growth factor receptormutant lung adenocarcinomas. Eur Respir Rev 2014; 23: 356-366.
\end{abstract}

Received: May 232014 | Accepted after revision: July 012014

J. Menis received a fellowship supported by Fonds Cancer (FOCA; Brussels, Belgium).

Conflict of interest: None declared.

Provenance: Submitted article, peer reviewed.

Copyright OERS 2014. ERR articles are open access and distributed under the terms of the Creative Commons Attribution Non-Commercial Licence 4.0. 


\section{Introduction}

In the past decade, progress in tumour biology, genomics technology, computational analysis and drug discovery has propelled advances in both translational and clinical cancer research. Despite the presence of other genomic and epigenetic alterations, many tumours are highly dependent on the function of a single oncogene for proliferation and survival. This concept is the so-called "oncogene addiction", where the signalling network becomes sufficiently unstable in the presence of a mutated oncoprotein that the latter plays an essential role in the malignant setting. Many genomic alterations create a deregulated signalling cascade; thus, the derivative mutant proteins are potential foci for targeted anticancer therapies, and biomarkers can be accurate predictors of their efficacy [1-3]. Lung cancer, among all thoracic malignancies, has become the prototype for genetically tailored targeted therapy. Nevertheless, the validation of biomarkers is currently affected by severe challenges, such as the multitude of assessment methods (i.e. immunohistochemistry, fluorescence in situ hybridisation, next-generation sequencing, etc.), reliability in terms of sensitivity and specificity, reproducibility of the test, feasibility of obtaining an adequate and representative tumour sample and, finally, the overall related costs.

Moreover, classical phase I, II and III models for drug development require huge resources in terms of large populations, infrastructure and financial resources; thus, limiting the number of experimental agents that can be tested and making it inefficient for the evaluation of targeted agents (i.e. overall high failure rates) $[4,5]$.

The average time for clinical trial development and new drug registration is reported to be $\sim 7.6$ years for oncological compounds, which is comparable to 7.8 years for cardiovascular compounds, but significantly longer compared to antiretrovirals (5.1 years) [6]. Therefore, there is an urgent need to streamline the development of new compounds. Finding a new balance between stakeholders also enhances the roles of molecular biologists, patients and payers. The overall goal is to define "trials designed to learn", which should then lead to subsequent "trials designed to conclude" [4]. As a matter of fact, in the genomics era, the main goal should be to identify large and meaningful differences in small molecularly selected groups of patients [7].

Clinical trials designed to enrich a target population by a predictive biomarker or statistically powered to prospectively evaluate a biomarker as co-primary or secondary end-points will most probably demonstrate a benefit to patient subgroups [8]. The main requirements are an adequate amount of tissue for molecular profiling, early co-development of biomarkers and targeted agents, and recognition of intratumour heterogeneity. Table 1 summarises the main study designs for predictive marker validation as described by MANDREKar and SARGENT [9].

Two main enrichment strategies can be used to avoid over-treatment and save valuable resources, by matching the right drug to the right subgroup of patients. They can be defined as: basket trials and umbrella trials.

TABLE 1 Main study designs for predictive marker validation

Unselected or all-comers

Sequential testing strategy

Marker by treatment interaction

Marker-based strategy

Targeted or enrichment

Hybrid
The compound is first tested in the overall population and then in the marker-defined subpopulation or vice versa, according to the strength of preliminary data

The marker is used as a stratification factor upfront: the overall study population is divided into two groups according to the marker status and each subpopulation is equally randomised to the experimental versus control arm

Patients are randomly assigned to receive their treatment either based on the biomarker status or not; thus, determining a significant overlap of patients treated with the same regimen in both the biomarker-based and non-biomarker-based arm

All patients are screened for molecular alteration but only the subpopulation who either express a specific molecular alteration or not are enrolled in the clinical trial to assess the safety, tolerability and clinical benefit of a specific treatment

This design requires well-established assay reproducibility and accuracy and early evidence of benefit

Only the marker-defined subgroup is randomly assigned to have the treatment based on that marker, the others subgroups are assigned to the standard of care

It is similar to the enrichment design but also includes the non-marker-defined subgroup; thus, being the preferable choice when there is prior evidence of marker-based treatment 
Planning for such types of studies is particularly challenging due to the limited available information in the literature describing the targeted sub-populations, which sometimes leads to overlap between such design types.

In order to overcome such limitations, new methodological strategies have been developed and will be described herein, including: new design strategies, such as the adaptive designs; basket and umbrella trials; and newly defined measures to assess and determine the efficacy of treatments, such as new end-points and imaging techniques.

\section{Adaptive design}

Clinicians continuously learn from their patients, applying knowledge gained from one set of patients to their forthcoming patients. This form of "adaptive" medicine has its statistical counterpart. In adaptive designs, modifications of some aspects of the trial can be prospectively planned so that changes ("adaptations") may take place while the study is ongoing, for example: a treatment arm or a subgroup of patients could be dropped; dose levels could be altered; and the trial size could be increased, if the tested compound proved to be less effective than expected at the interim analyses.

The main goal of adaptive-design trials is to learn and address several hypotheses, in order to improve the accuracy of the study itself and speed up the development of the compound [12]. The most frequently used adaptive designs are summarised in table 2 [9, 12-14]. Adaptive designs are increasingly being used as there is growing acceptance and general encouragement from regulators (e.g. US Food and Drug Administration (FDA) guidance) [15]. These regulators have approved some compounds on the basis of phase I and II studies that adopted enrichment strategies or expansion cohort strategies as a consequence of recruitment difficulties in rare types of tumour (e.g. vismodegib and crizotinib [16, 17]).

Nevertheless, it takes more time to plan an adaptive design trial as it actually involves all stakeholders, i.e. clinicians, IT personal, project managers, drug providers and biostatistician. IT personnel need to organise frequent intake of data and large flows of information. Rigorous analysis and astute clinical interpretation of comprehensive genomic data is impossible without the assistance of computational algorithms to support clinical-grade data interpretation. Project managers need to organise flexible drug supply and only a handful of biostatisticians have extensive training in this area; many of them still consider some subjectivity in the design [18]. Moreover, adaptive randomisation might worsen the precision of treatment effect estimates by increasing variability, mainly owing to unequal subject allocation in the treatment arms [19].

Adaptive designs may provide a more distinct advantage in the study of novel drugs that do not have a clearly understood mechanism of action, or in situations when the biological characteristics of the target are uncertain. In these situations, real-time, complex biomarker analyses might accelerate the identification and further testing of potential biomarker-therapy relationships. Conversely, when hypotheses about the drug target and its biological features are well-understood, it may be less efficient than either prospective biomarker-directed trials addressing mature hypotheses or conventional-targeted therapy studies in less restricted patient populations that incorporate retrospective analyses of well-defined biomarkers [12].

The main concerns with these designs are: 1) the control of type I error rate; 2) the minimisation of impact of any adaptation-associated statistical or operational bias on the estimates of treatment effects; and 3) the real interpretability of the results. Therefore, comprehensive simulations are usually required to understand the design characteristics and properly estimate type I and II error rates during the planning stage; these may pose computational challenges if the model is complex [13].

Adaptive designs also fit well with basket and umbrella studies, which represent novel approaches for targeted therapeutics testing. Both types of studies have the potential to accelerate the drug development process so that the correct therapies can be delivered quickly to the right patients.

\section{Basket trial}

This type of study design allows patients with multiple diseases and one or more target to be enrolled in cohorts or groups in one trial (the basket) (fig. 1). This allows researchers to separately analyse the responses of patients as each tumour type can be put in one cohort, and assess the impact of the drug on all of the patients as one group. If one group shows a good response, we expand this group to immediately assess whether others could benefit from the new therapy. If another group does not show evidence of effectiveness, this group may be closed and the other cohort can continue the recruitment [19, 20].

There are several available examples for basket trials, which can be grouped into three main categories depending on the mechanism of action of the drug (inhibition of one target versus multiple targets) and the molecular selection of the patients. 


\section{One drug, several tumour types}

These studies investigate one compound that targets several tumour types. An example is the ongoing phase II study investigating the use of PHA-739358 administered by a 24-h intravenous infusion every 14 days in advanced/metastatic breast, ovarian, colorectal, pancreatic, small cell lung cancer and nonsmall cell lung cancers (NSCLC) [21]. Merging parallel phase II studies into a single trial simplifies the operational aspects, but implies to open centres that treat different types of cancer in order to be able to recruit most, or all, the populations of the study arms.

\section{One drug, one molecular alteration, several tumour types}

These studies investigate one compound that targets one specific molecular alteration in several tumour types, two such examples are as follows.

Imatinib mesylate was approved by the US FDA and European Medicines Agency for use on the basis of a single phase II trial basket study of patients with non-gastrointestinal stromal tumour malignancies and Kit mutations. The study population included patients suffering from myelodysplastic or myeloproliferative syndrome diseases, aggressive systemic mastocytosis, hypereosinophilic syndrome/chronic eosinophilic leukaemia and dermatofibrosarcoma protuberans. All these diseases were associated with Abl, Kit or platelet-derived growth factor receptor protein tyrosine kinases. The primary objective was to identify evidence of imatinib activity with tumour response as the primary end-point [22].

Another good example of this study design is study with vemurafenib targeting $B R A F^{\mathrm{V} 600 \mathrm{E}}$-mutated tumours (fig. 1). BRAF mutations have been identified in $\sim 8 \%$ of all human cancers; however, the prevalence is highly variable with a high frequency being present in melanoma, thyroid carcinoma and colorectal cancer [23]. The study screened for the $B R A F^{\mathrm{V} 600 \mathrm{E}}$ mutation in patients who were diagnosed with a malignancy (excluding melanoma and papillary thyroid cancer) or multiple myeloma refractory to standard therapy, or for whom standard or curative therapy did not exist or was not considered appropriate by the investigator [10].

\section{One drug, several molecular alterations, several tumour types}

These studies investigate one compound that targets several molecular alterations in several tumour types, two such examples are as follows.

The first example is the French National AcSé program, which can be considered a large-scale basket trial. It is a biology driven, trans-tumoural, multicentric, phase II study assessing the efficacy and safety of crizotinib as monotherapy in 23 cohorts of patients with at least one alteration between anaplastic lymphoma kinase $(A L K), M E T, R O N$ or ROS1. A cohort is defined by a histopathology and crizotinib-targeted alteration (e.g. gastric cancer with MET amplification). Among the included tumour types were several sarcoma tumours, gastrointestinal tumours (gastric, colorectal, hepatocarcinoma and cholangiocarcinoma), NSCLC, breast cancer, kidney cancer, ovarian cancer, thyroid cancer, glioblastoma and anaplastic large cell lymphoma. For each cohort, a two-stage design is implemented. Three different statistical designs are considered a priori according to the expected response rate and incidence. The study is currently recruiting patients [24]. Given the number of centres (up to 250), this can be viewed as an expanded access programme with a rigorous exploration of the safety and efficacy profile.
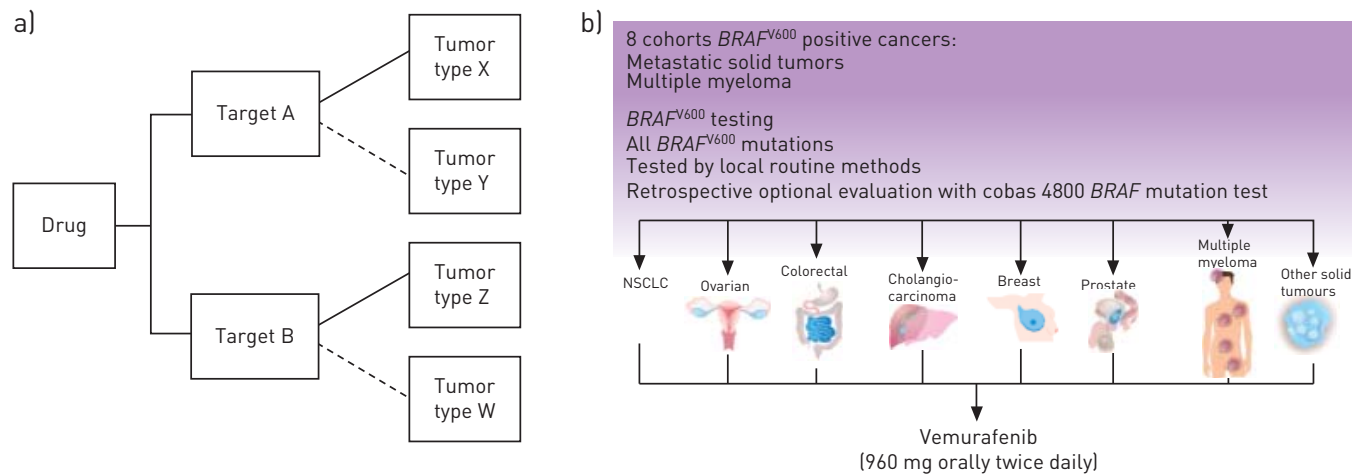

FIGURE 1 a) Schematic example of a basket trial. These trials are often viewed as parallel, phase II trials for one drug, but within the same legal entity on the basis of a common denominator that can be molecular alteration(s). Most basket trials are histology-independent and aberration-specific clinical trials if a predictive marker is used. These trials are usually tested locally and often fall into the "trial to learn" category. b) Schematic illustration of the Vemurafenib basket trial [10]. NSCLC: nonsmall cell lung cancer. Images courtesy of Gustave Roussy Cancer Campus Grand Paris, Villejuif, France. 
TABLE 2 Summary of the most common types of adaptive designs

Type of design

Adaptive randomisation

Adaptive group sequential

Flexible sample size re-estimation

Drop the losers (or picking up the winners)

\section{Adaptive dose-finding}

Biomarker adaptive

\section{Adaptive treatment-switching}

\section{Adaptive hypothesis}

Phase I/II (or II/III) adaptive seamless trial

\section{Description}

Allows modification of the randomisation schedule based on treatment, response, etc.

The goal is to assign more patients to a promising test treatment

Patient characteristics should be balanced between the groups

Not feasible for a large trial with relatively long treatment duration

Classical group sequential design with pre-specified interim analyses

Options of additional adaptations include:

Sample size re-estimation

Modification/deletion/addition of treatment arms

Change of study end-points

Modification of dose, schedule and treatment duration

Allows for sample size adjustment/re-estimation based on the observed interim data

May be biased as the original power analysis for sample size calculation is performed prior to conducting the study

The observed interim difference is based on a small number of subjects and:

May not be statistically significant (maybe due to chance alone)

May not be reproducible

Can be either based on overall data or based on criteria such as treatment-effect size, conditional power and/or reproducibility

The US Food and Drug Administration recommends re-estimation methods be based on interim analyses on overall data

A multistage design that allows:

Dropping the inferior treatment groups

Modifying treatment arms

Adding additional arms after the review of interim data

Risks related to this study design are:

Not having enough statistical power at the end of the first stage

The investigator may pick up the wrong group

Used in early phase clinical development to identify the maximum tolerated dose, as it is desirable to achieve it with a limited number of patients

The selection of initial dose, dose range and criteria for dose escalation and/or dose de-escalation is important for the success of the trial

A recently proposed new method (the continual reassessment method) allows continual reassessment of the dose-response relationship based on the accumulative data collected

Enables adaptations based on the response of biomarkers

Involves biomarker qualification, optimal screening design, and model selection and validation

It is useful to identify:

The patient population most likely to respond

The natural course of disease

Early disease

Allows a patient's treatment to be switched from one assignment to an alternative if there is evidence of lack of efficacy, disease progression or safety issues

Permits changes in hypotheses in response to interim analysis results to achieve the desired power Some examples include pre-planned switching:

From a single hypothesis to a composite or multiple hypotheses

Between the null hypothesis and the alternative hypothesis

Between the primary and the secondary end-points

It combines the objectives, traditionally addressed in separate trials, into a single study

The most common examples include:

Adaptive seamless phase $\mathrm{I} / \mathrm{II}$ design

Adaptive seamless phase II/III design

The second example is the ongoing multi-tumour CREATE (Cross-tumoral Phase 2 with Crizotinib) trial [25], which explores treatment with crizotinib in patients with advanced disease across six heterogeneous malignancies that are, in part, associated with $A L K$ and/or MET alterations. Each tumour type constitutes a study cohort and each cohort has two sub-cohorts for each molecular subtype (ALK/MET ${ }^{+}$and ALK/MET ${ }^{-}$patients) [25].

\section{Umbrella trials}

Unlike basket studies, where researchers test the effect of a single drug on a single mutation (usually tested locally) in a variety of cancer types, "umbrella" trials are designed to test the impact of different drugs on 
different mutations in a single type of cancer, on the basis of a centralised molecular portrait performed after obtaining informed consent: one disease, several molecular subtypes, several therapies. This design allows validation of a strategy based on a mixture of biomarkers and drugs (fig. 2).

Umbrella studies require a strong collaboration to be in place, as a consistent molecular profile and harmonisation of the cohorts for each biopsy, assay and medication are needed. In addition, a solid collaboration between academia and industry is required.

An example of a successful umbrella trial is the BATTLE (Biomarker-integrated Approaches of Targeted Therapy for Lung Cancer Elimination) trial [26]. The BATTLE trial was the first prospective, biopsymandated, biomarker-based, adaptively randomised study in NSCLC. 255 pre-treated NSCLC patients were adaptively randomised to erlotinib, vandetanib, erlotinib plus bexarotene, or sorafenib, based on relevant molecular biomarkers analysed in fresh core needle biopsy specimens. The BATTLE study establishes the feasibility of "real-time" biopsies, taking a substantial step towards realising personalised lung cancer therapy by integrating real-time molecular laboratory findings in delineating specific patient populations for individualised treatment [26].

The study is characterised by two major phases. In the first phase, patients were randomly assigned in equal ratios to receive one of four treatments. In the second (adaptive) phase, treatments were randomly assigned based on the efficacy results of biomarker testing, i.e. treatment assignment decisions were still randomised but weighting was predicated on the molecular profiling results generated in real time [26]. BATTLE-2, a phase II, randomised, multi-arm study in advanced NSCLC, is currently ongoing and has been built on the experience of the previous study [27].

SAFIR02_Lung (Intergroup Trial UNICANCER UC 0105-1305/IFCT 1301) is an open-label, multicentric, randomised, phase II trial using high-throughput genome analysis for therapeutic decision making [11]. Patients with advanced NSCLC are re-biopsied during the two initial platinum-based chemotherapy cycles. A high-throughput molecular analysis (comparative genomic hybridisation (CGH) array, next-generation sequencing) is performed and analysed during the two subsequent cycles. Patients with a molecular alteration are then randomised between a standard maintenance treatment or a molecular-driven maintenance treatment among six targeted therapies (fig. 2). The study aims to evaluate whether treatment with targeted agents guided by high-throughput molecular analysis (CGH array, next-generation sequencing) improves progression-free survival (PFS; the primary end-point) compared to standard maintenance [11].

Arm A: Treatment assigned according to the presence of a molecular abnormality

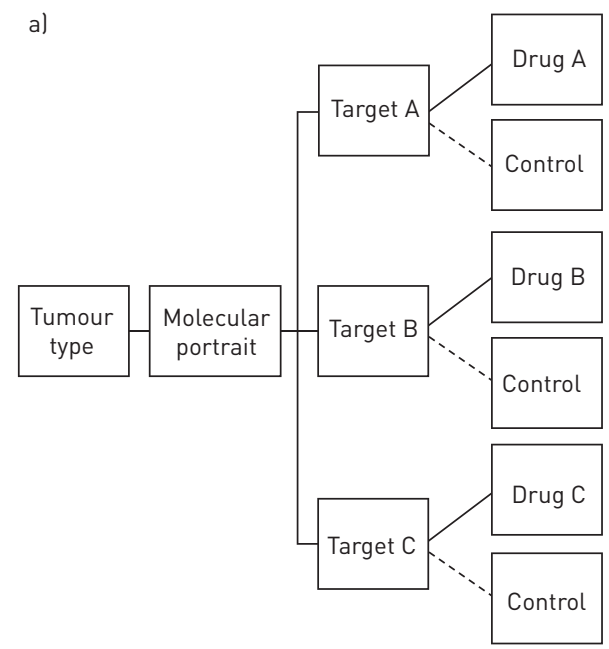

b)

Tumour biopsy:

Next-generation sequencing

(50 genes, ampliSeq, ion torrent) CGH array

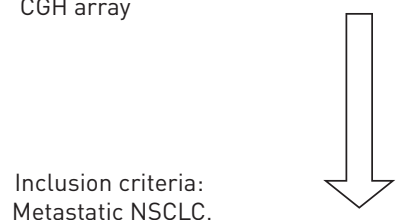

Metastatic NSCLC, no active EGFR mutation or $A L K$ translocation

chemonaïve or on first-line platinum-based chemotherapy (maximum 2 cycles) $\mathrm{n}=650$

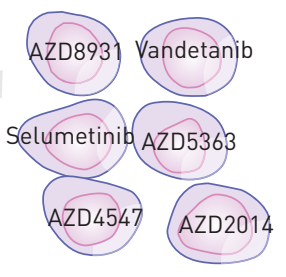

Arm B: chemotherapy not guided by any molecular abnormality

Pemetrexed

(nonsquamous Erlotinib NSCLCl (squamous NSCLC)

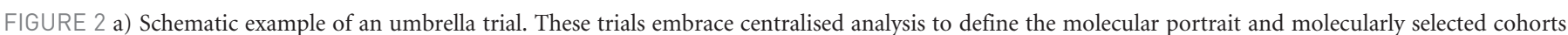

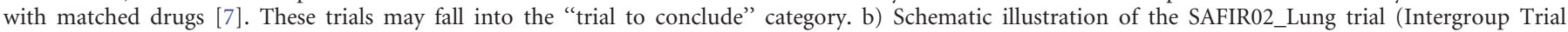
UNICANCER UC 0105-1305/IFCT 1301). This is an open-label, multicentric, randomised, phase II trial [11]. NSCLC: nonsmall cell lung cancer; EGFR: epidermal growth factor receptor; ALK: anaplastic lymphoma kinase; CGH: comparative genomic hybridisation. Images courtesy of Gustave Roussy Cancer Campus Grand Paris, Villejuif, France. 
Another umbrella trial is the WINTHER (A Study to Select Rational Therapeutics Based on the Analysis of Matched Tumor and Normal Biopsies in Subjects with Advanced Malignancies) study. This study selects rational therapeutics based on the analysis of matched tumour and normal biopsies in subjects with advanced malignancies [28]. It is an academic, international, nonrandomised molecular triage that includes patients with any metastatic solid tumour type who have received at least one prior treatment and are able to undergo biopsy. The main objective of the study is to compare the PFS of the current treatment versus the previously prescribed treatment: i.e. the PFS ratio. The therapeutic decision will be based on an estimated drug efficacy scoring bioinformatics tool, which integrates all data on biomarkers, molecular aberrations, targeted and non-targeted therapies, and predictive algorithms of efficacy. The study is currently recruiting in France, Spain, the USA, Canada and Israel; the aim is to recruit 200 patients in total [28].

Finally, the MATCH (Molecular Analysis for Therapy Choice) trial is an umbrella protocol for multiple single-arm, phase II trials. Approximately 3000 patients will be screened, with 800-1000 participants being enrolled: $25 \%$ of whom will have rare cancers, while $75 \%$ will have common cancers including breast, lung, colon or prostate cancer. Whole-genome sequencing will be performed on available tissue from the enrolled patients to identify 100 actionable mutations. Co-primary end-points are overall response rate and PFS rate at 6 months [29].

\section{End-point}

At present, identifying an end-point that truly represents an indication of compound effectiveness is still a subject of debate in randomised clinical trials. Choosing the most appropriate end-point (i.e. measure of the study objective) is among one of the most necessary factors contributing to effective benefit measurement of investigational treatment.

In the 1970s, the US FDA approved some therapeutic agents based on studies with overall response rate as the primary end-point. However, overall response rate is not a direct measure of benefit.

Overall survival is calculated from the time of randomisation to the time of death and has been the most frequently used primary end-point in lung cancer clinical trials, due to its objectiveness. It is classically and universally accepted by regulatory agencies to provide approval for cancer treatment, as it is precise and easy to measure.

However, in other studies, overall survival might not always be the optimal end-point of choice as it includes non-cancer deaths and might be affected by crossover [30]. At the time when there were no molecular alterations and only one approved chemotherapy line, overall survival was an accurate end-point as it reflected tumour shrinkage and time to progression. One example is the Eastern Cooperative Oncology Group study, which compared four different types of first-line chemotherapy regimens and showed no difference in overall response rate, time to progression and overall survival [31]. With the advance of drug development driven by progress in molecular biology, several lines of treatment are now possible, even in the advanced disease setting. This implies that patients may receive more treatment at the time of progression that could potentially obscure overall survival. Therefore, the use of overall survival as the sole end-point may no longer be the case in this setting.

Symptom end-points could be alternative clinically meaningful end-points as they reflect the patient's perspective but such end-points suffer due to the difficulty of real blinding, missing data, lack of validated tools and the difficulty of assessing the clinical significance of small changes. New proposals are under investigation, such as the combination of PFS with quality of life assessment, which is particularly meaningful in lung cancer patients [32]. Recently, more and more agents have been approved with an accelerated procedure based on early read-out clinical end-points or surrogate end-points $[4,7]$.

A surrogate end-point is an end-point intended to replace a clinical end-point of interest that is difficult to observe in a trial, a variable that provides an indirect measurement of the effect in situations where direct measurement of clinical effect is not feasible or practical [33]. Prentice's criteria state that to be a direct substitute for a clinical benefit end-point, the surrogate end-point must be correlated with the clinical outcome and must also fully capture the net effect of treatment on the clinical outcome [34]. A surrogate end-point can be used if accurate, reliable, reproducible, sensitive and specific [35].

Despite some pitfalls in validating its surrogacy [36], as a strong association between a surrogate and a true end-point and between treatment effect on the surrogate and true end-point need to be proved, PFS has started to be used more frequently in clinical trials as a primary end-point. For locally advanced NSCLC stages, PFS, as a direct measure of the treatment effect on the tumour burden, tumour shrinkage and stabilisation, was hypothesised as a surrogate end-point for overall survival with the aim of both avoiding the bias related to crossover and/or subsequent treatment and of obtaining an earlier assessment of efficacy $[30,37]$. Recently, disease-free survival and PFS have been proved to be valid surrogate end-points in an 
individual patient data analysis of six meta-analyses of 60 randomised studies for earlier stages and locally advanced disease, respectively [37].

Although PFS has been validated as a surrogate end-point for locally advanced disease, its use in advanced disease is still controversial. The standard assumption in determining sample size in a clinical trial is that the hazard ratio associated with treatment is constant over time, but this assumption has no empirical justification. Indeed, the hazard ratio of death is likely to change after disease progression as PFS differs among the different treatment lines.

This last concept and the limited value of overall survival as a primary end-point have been particularly emphasised in trials studying the use of targeted agents. As a matter, of fact all six randomised studies with epidermal growth factor receptor (EGFR) tyrosine kinase inhibitors (TKIs) in first-line advanced NSCLC have met their primary end-point demonstrating a significant PFS benefit, but did not show any overall survival improvement despite a hazard ratio of $\sim 0.5$ for PFS [3, 38-43].

Overall survival is the classical end-point, but powering a trial to show an overall survival benefit can be challenging as it requires a large number of patients and consequently some trade-offs, including: slowing drug development; increasing the cost of medical care; and using patient resources that might be better allocated to investigating other therapies. Moreover, the identification of molecular alterations has led to the definition of subpopulations that can be limited in numbers; some mutations have a prevalence of $1-2 \%$ and powering studies for overall survival in such a limited population is particularly challenging.

Survival post-progression (SPP) is a time-to-event measure that can be analysed by using standard methods of survival analysis and, if combined with PFS, provides the overall survival of a patient (OS $=$ PFS + SPP). In general, the longer the SPP, the lower the chance that PFS and overall survival correlate [44].

At disease progression, there are a variety of choices for the patient and the physician to make: 1) continue the same regimen; 2) cross over to the other arm; 3) switch to another treatment (e.g. next-generation inhibitors); or 4) no treatment at all, but commonly this choice is not randomised and therefore not comparable between the study arms. For example, a study on a series of ALK-positive NSCLC patients treated with crizotinib showed that, according to RECIST (Response Evaluation Criteria In Solid Tumors) 1.1, continuing crizotinib beyond disease progression resulted in a significant prolongation of the SPP and consequently of overall survival [45].

An end-point that is currently used in several previously mentioned studies (MOSCATO and WINTHER) is the PFS ratio calculated between the PFS of the therapy selected by a patients tumour's molecular profiling (b) with the PFS of the most recent prior therapy on which the patient has just experienced progression (a). If the ratio $(b / a)$ is $\geqslant 1.3$, then the molecular selected therapy can be considered beneficial for the patient [46].

In addition, calculating the time between randomisation and disease progression, not of the first treatment but the disease progression after the subsequent treatment, could be an interesting end-point as it would take into account the influence of the treatment under investigation on the following treatment line. Finally, attention should be paid to the development of new end-points based on functional imaging, tumour kinetics and the validation of predictive markers.

\section{Imaging}

To date, computed tomography (CT) and magnetic resonance imaging (MRI) have been the most accurate techniques, both in terms of sensitivity and specificity, for tumour response assessment, particularly for thoracic malignancies. As a result, they are recommended by the RECIST criteria.

Functional imaging was developed with the aim of providing additional information as it is able to visualise heterogeneous metabolic processes; techniques include: 2-fluro-2-deoxy-D-glucose (FDG)-positron emission tomography (PET)/CT, $\left[{ }^{18} \mathrm{~F}\right]$ fluorothymidine (FLT)-PET, dynamic contrast enhancing MRI and diffusion-weighted MRI.

FDG-PET/CT is a well-documented and widely used imaging modality for the diagnosis and staging of lung cancer patients due to its higher sensitivity and specificity for lymph node and metastasis detection. It is also increasingly used for the assessment of treatment effects during and after chemotherapy. However, PET is not an accepted surrogate technique for the assessment of response rate in clinical trials [47], and standard CT and MRI are still the most commonly used imaging techniques. Nevertheless, several studies have shown that it has added value in outcome prediction for both standard chemotherapy and EGFR TKIs, as well as in assessing early response [48].

HACHEMI et al. [49] evaluated whether $\left[{ }^{18} \mathrm{~F}\right] \mathrm{FDG}-\mathrm{PET} / \mathrm{CT}$, performed within 2 weeks of starting erlotinib, could predict tumour response defined by RECIST 1.1 criteria after 8 weeks of treatment in patients with 
stage IIIA-IV NSCLC. A $\left[{ }^{18} \mathrm{~F}\right]$ FDG-PET/CT scan within 2 weeks after starting erlotinib therapy could predict response, PFS and overall survival [49]. Despite the success of PET/CT in oncology practice, it has innate limitations in terms of soft tissue contrast resolution and radiation exposure.

The idea of combining PET and MRI has garnered much attention, as it is a combination of molecular information offered by PET and anatomic/potentially functional information with high contrast and spatial resolution provided by MRI. The main advantages are increased resolution, better assessment and characterisation of brain and liver metastases, good soft tissue contrast and minor radiation dose. However, this technique is costly and time consuming, and the interpretation of its results is not yet commonly agreed [50].

FLT-PET is based on the intracellular trapping of the tracer fluorothymidine by thymidine kinase-1, thus, reflecting proliferation. This technique has been widely investigated, although only in small series. FLT-PET uptake has been investigated as a marker for prognostic stratification of advanced NSCLC patients treated with EGFR-TKIs [51], but also as a predictor of early response and relapse [52, 53]. More recently, it has also been investigated to assess response to chemoradiotherapy with promising results, although in very small patient cohorts [54, 55].

Diffusion-weighted MRI depends on the microscopic mobility of water in tissues, and has been used to evaluate tumour microstructure that reflects tumour aggressiveness and response to treatment [56]. The advent of anti-angiogenic drugs in cancer therapy necessitates an imaging modality that can longitudinally assess post-treatment changes in tumour vasculature.

Real-time sonography during contrast administration (dynamic contrast enhancement) requires Doppler ultrasound and a microbubble contrast agent, which enhances the vessel signal and enables blood vessels with a diameter of $40 \mu \mathrm{m}$ to be detected.

Dynamic contrast enhancing ultrasound provides both morphological and functional perfusion data [57]. It helps evaluate lymph node vascularity and perfusion, and might help in assessing early tumour response $[58,59]$.

Dynamic contrast enhancing ultrasound is supported by the French National Cancer Institute, which is currently studying this technique in metastatic cancers in order to establish optimal perfusion parameters and timing for quantitative anticancer efficacy assessments [60].

Another new technique that has been developed with the aim of better characterising the tumour vasculature and angiogenesis is perfusion tomography. This technique assesses the temporal changes in tissue attenuation after the intravenous administration of iodinated contrast media. Perfusion tomography has proved to be useful in monitoring response to both chemotherapeutics and targeted agents, identifying tumour heterogeneity and better characterising lesions; e.g. in inflammatory condition cases. However, it has also some limitations such as acquisition effects that are mainly related to motion artefacts (including breathing) and technical reproducibility and variability [61, 62].

\section{Future challenges: development of immunotherapeutic agents}

Immunotherapy of cancer has entered a new and exciting phase because of the discovery of checkpoint receptor inhibitors, such as cytotoxic T-lymphocyte antigen-4 (CTLA-4) and, more recently, programmed death-1 (PD1) receptor and its ligand. As a matter of fact, antibodies targeting PD1 receptors/PD1 ligands appear to be a breakthrough treatment in terms of clinical impact on one or more tumours, on the basis of clinical evidence that was sufficiently strong enough to allow the accolade of "drug of the year" [63].

Two PD-1 receptors (nivolumab and lambrolizumab) and three PD1 ligands (BMS-936559, MPDL3280A and Medi-4736) have been investigated in phase I studies and are under further development in NSCLC [64]. In all the phase I trials, the maximum tolerated dose was not reached, and all doses were found to be safe. Responses have been confirmed and range from $8 \%$ to $25 \%$, and are often higher in the squamous subpopulation $(\sim 10-35 \%)$. We have no mature data on PFS but it is likely to greater than 1 year in responders.

The frequency of immune-related toxicities from anti-PD1 receptor/ligand treatment is definitely less than that from anti-CTLA-4 treatment, with the exception of pneumonitis. The common drug-related adverse reactions were decreased appetite, anaemia, diarrhoea, nausea, pruritus, fatigue, pneumonitis and elevated transaminase [65-68]. Among the next steps in the further development of these compounds is the identification of predictive subtypes that will respond better to immune-checkpoint blockade.

In the MPDL-3280A phase I trial, PD1 ligand-positive tumours showed an overall response rate of $80 \%$ versus $14 \%$ for the PD1 ligand-negative tumours [67]. The combination of this class of compounds with local and/or systemic treatments is also among the future challenges for their development. 
Meanwhile, the identification of the best criteria to assess response is also a matter of discussion. It is well known that objective responses defined by the World Health Organization (WHO) or RECIST are infrequent with immunotherapy. Finding the balance between criteria that would avoid underestimating the antitumor activity of the immunotherapy agent and criteria that would lead to an overestimation is a delicate, but challenging, task [69]. As a matter of fact, in a study investigating a CTL-4A inhibitor, i.e. ipilimumab, different patterns of tumour response were described [70].

Among the most difficult tumour response pattern clinicians face is the "tumour flare", which has been described as an initial increase in the tumour burden followed by tumour shrinkage. Recognising tumour flare and differentiating it from true treatment failure (disease progression) is key for clinicians and requires both training and development of new tools merged with the clinical insight brought by the patients (modification of performance status and/or symptoms).

Immune-related response criteria have been proposed by a collaborative group of $\sim 200$ oncologists, immunotherapists, and regulatory experts on the basis of clinical observations. For the immune-related response criteria only index and measurable new lesions are taken into account and antitumor response is based on the total measurable tumour burden [71]. However these criteria still need to be validated and should be further investigated in studies on checkpoint inhibitors.

\section{Conclusions}

Personalised therapy is likely to increase the effectiveness of patient's treatment, in both a clinically meaningful and cost-effective way once the outstanding hurdles in clinical trial methodology are overcome.

The current challenges for researchers include: determining whether similar aberrations in different histologies (cross-cancer similarity) have a comparable biological significance and can be successfully targeted with the same agents; the presence of mutations of unknown functionality; intra-patient heterogeneity and clonal evolution; the modulation of a target during a clinical trial and/or paradoxical activation of a survival pathway; and logistics (drug supply, rapid turnaround time and large scale size needing intergroup collaborations).

For example, in the case of disease progression, the type of disease progression during treatment with targeted agents is different from the one commonly experienced with chemotherapeutics. Disease progression can be oligo-disease progression (i.e. less than four sites), systemic-disease progression (in more than four sites), or central nervous system disease progression (occurring only in the central nervous system). All these types of disease progression can (and should) be treated with different strategic options [72]. Moreover, an increasing number of genetic abnormalities have been identified; the Lung Cancer Consortium has identified actionable molecular abnormalities in 54\% of cases of lung adenocarcinoma [73].

Costs for sequencing are decreasing and are becoming affordable for single investigators [74, 75]; however, the prevalence of the identified molecular alterations is so low in most cases that designing clinical trials in these rare populations is extremely challenging for both academia and pharmaceutical companies.

Therefore, the European Organization for the Research and Treatment of Cancer has undertaken a joint effort in collaboration with the European Thoracic Oncology Platform and has developed SPECTAlung (Screening Program for Efficient Clinical Trial Access for patients with lung cancer). This platform aims at screening patients with any thoracic malignancy at any stage in 15 selected highly specialised and qualified thoracic centres in Europe and offering them access to downstream clinical trials. This project is built on the awareness that creating meaningful data, also through innovative designs, for reimbursement strategies is and will be a major goal of future clinical trials.

\section{References}

1 Buettner R, Wolf J, Thomas RK. Lessons learned from lung cancer genomics: the emerging concept of individualized diagnostics and treatment. J Clin Oncol 2013; 31: 1858-1865.

Sculier JP. Nonsmall cell lung cancer. Eur Respir Rev 2013; 22: 33-36.

Sebastian M, Schmittel A, Reck M. First-line treatment of EGFR-mutated nonsmall cell lung cancer: critical review on study methodology. Eur Respir Rev 2014; 23: 92-105.

4 Burock S, Mounier F, Lacombe D. How can innovative forms of clinical research contribute to deliver affordable cancer care in an evolving health care environment? Eur J Cancer 2013; 49: 2777-2783.

5 Freidlin B, Korn EL, Gray R, et al. Multi-arm clinical trials of new agents: some design considerations. Clin Cancer Res 2008; 14: 4368-4371.

6 Kaitin KI, DiMasi JA. Pharmaceutical innovation in the 21st century: new drug approvals in the first decade, 2000-2009. Clin Pharmacol Ther 2011; 89: 183-188.

7 Sleiifer S, Bogaerts J, Siu LL. Designing transformative clinical trials in the cancer genome era. J Clin Oncol 2013; 31: 1834-1841.

8 Gandara DR, Li T, Lara PN Jr, et al. Algorithm for codevelopment of new drug-predictive biomarker combinations: accounting for inter- and intrapatient tumor heterogeneity. Clin Lung Cancer 2012; 13: 321-325. 
9 Mandrekar SJ, Sargent DJ. Clinical trial designs for predictive biomarker validation: theoretical considerations and practical challenges. J Clin Oncol 2009; 27: 4027-4023.

10 Clinicaltrials.gov. A Screening Study to Detect BRAF V600 Mutation-Positive Patients for Enrollment into Clinical Research Studies of Zelboraf (Vemurafenib). NCT01804140. http://clinicaltrials.gov/ct2/show/NCT01804140?term= NCT01804140\&rank=1 Date last updated: July 7, 2014. Date last accessed: July 2014.

11 Clinicaltrials.gov. Intergroup Trial UNICANCER UC 0105-1305/ IFCT 1301: Efficacy of Targeted Drugs Guided by Genomic Profils in Metastatic NSCLC Patients (SAFIR02_Lung). NCT02117167. http://clinicaltrials.gov/ct2/show/ NCT02117167?term=NCT02117167\&rank=1 Date last updated: April 28, 2014. Date last accessed: April 2014.

12 Berry DA. Adaptive clinical trials in oncology. Nat Rev Clin Oncol 2012; 9: 199-207.

13 Chow SC. Adaptive clinical trial design. Annu Rev Med 2014; 65: 405-415.

14 Don G. A varying-stage adaptive phase II/III clinical trial design. Stat Med 2014; 33: 1272-1287.

15 European Medicines Agency. Guideline on the evaluation of anticancer medicinal products in man. www.ema. europa.eu/docs/en_GB/document_library/Scientific_guideline/2013/01/WC500137128.pdf Date last updated: December 13, 2012. Date last accessed: January 11, 2013.

16 Von Hoff DD, LoRusso PM, Rudin CM, et al. Inhibition of the hedgehog pathway in advanced basal-cell carcinoma. N Engl J Med 2009; 361: 1164-1172.

17 Kwak EL, Bang YJ, Camidge R, et al. Anaplastic lymphoma kinase inhibition in non small cell lung cancer. N Engl J Med 2010; 363: 1693-1703.

18 Van Allen EM, Wagle N, Levy MA. Clinical analysis and interpretation of cancer genome data. J Clin Oncol 2013; 31: 1825-1833.

19 Thall PF, Wathen JK. Practical Bayesian adaptive randomization in clinical trials. Eur J Cancer 2007; 43: 859-866.

20 Willyard C. "Basket studies" will hold intricate data for cancer drug approvals. Nat Med 2013; 19: 655.

21 EU Clinical Trials Register. Phase II study of PHA-739358 administered by a 24-hour IV infusion every 14 days in advanced/metastatic breast, ovarian, colorectal, pancreatic, small cell lung and non small cell lung cancers. EudractCT Number 2006-003772-35 www.clinicaltrialsregister.eu/ctr-search/search?query=PHA-739358+ Date last updated: July 2014. Date last accessed: July 2014.

22 Heinrich MC, Joensuu H, Demetri GD, et al. Phase II, open-label study evaluating the activity of imatinib in treating life-threatening malignancies known to be associated with imatinib-sensitive tyrosine kinases. Clin Cancer Res 2008; 14: 2717-2725.

23 Davies H, Bignell GR, Cox C, et al. Mutations of the BRAF gene in human cancer. Nature 2002; 417: 949-954.

24 Clinicaltrials.gov. Phase 2 Study Assessing Efficacy and Safety of Crizotinib in Patients Harboring an Alteration on ALK, MET or ROS1 (AcSé). NCT02034981. http://clinicaltrials.gov/ct2/show/NCT02034981?term=NCT02034981\& rank=1 Date last updated: January 10, 2014. Date last accessed: January 2014.

25 Clinicaltrials.gov. CREATE: Cross-tumoral Phase 2 with Crizotinib. NCT01524926. http://clinicaltrials.gov/ct2/ show/NCT01524926?term = NCT01524926\&rank=1 Date last updated: June 2, 2014. Date last accessed: June 2014.

26 Kim ES, Herbst RS, Wistuba II, et al. The BATTLE trial: personalizing therapy for lung cancer. Cancer Discov 2011; 1: 44-53.

27 Clinicaltrials.gov. BATTLE-2 Program: a Biomarker-Integrated Targeted Therapy Study. NCT01248247. http:// clinicaltrials.gov/ct2/show/NCT01248247?term $=$ NCT01248247\&rank=1 Date last updated: May 30, 2014. Date last accessed: May 2014.

28 Clinicaltrials.gov. A Study to Select Rational Therapeutics Based on the Analysis of Matched Tumor and Normal Biopsies in Subjects With Advanced Malignancies (WINTHER). NCT01856296. http://clinicaltrials.gov/ct2/show/ NCT01856296?term $=$ NCT01856296\&rank=1 Date last accessed: January 2013.

29 National Cancer Institute. Molecular Analysis for Therapy Choice (NCI-MATCH). http://deainfo.nci.nih.gov/ advisory/ncab/164_1213/Conley.pdf Date last updated: December 2013. Date last accessed: December 2013.

30 Soria JC, Massard C, Le Chevalier T. Should progression-free survival be the primary measure of efficacy for advanced NSCLC therapy? Ann Oncol 2010; 21: 2324-2332.

31 Schiller JH, Harrington D, Belani CP, et al. Comparison of four chemotherapy regimens for advanced non-smallcell lung cancer. N Engl J Med 2002; 346: 92-98.

32 Schrimpf D, Manegold C, Pilz LR. Design of clinical studies: adaptive randomization and progression-free survival (PFS) as an endpoint in clinical studies of advanced non small cell lung cancer (NSCLC). Int J Clin Pharmacol and Ther 2013; 51: 84-86.

33 International conference on harmonisation of technical requirements for registration of pharmaceuticals for human use. ICH harmonised tripartite guideline. www.ich.org/fileadmin/Public_Web_Site/ICH_Products/Guidelines/ Efficacy/E9/Step4/E9_Guideline.pdf Date last updated: January 2014. Date last accessed: January 2014.

34 U.S. Food and Drug Administration. www.fda.gov Date last updated: March 7, 2014.

35 Temple R. Meta-analysis and epidemiologic studies in drug development and postmarketing surveillance. JAMA 1999; 281: 841-844.

36 Laporte S, Squifflet P, Baroux N, et al. Prediction of survival benefits from progression-free survival benefits in advanced non-small-cell lung cancer: evidence from a meta-analysis of 2334 patients from 5 randomised trials. BMJ Open 2013; 3.

37 Mauguen A, Pignon JP, Burdett S, et al. Surrogate endpoints for overall survival in chemotherapy and radiotherapy trials in operable and locally advanced lung cancer: a re-analysis of meta-analyses of individual patients' data. Lancet Oncol 2013; 14: 619-626.

38 Mok TS, Wu YL, Thongprasert S, et al. Gefitinib or carboplatin-paclitaxel in pulmonary adenocarcinoma. $N$ Engl J Med 2009; 361: 947-957.

39 Maemondo M, Inoue A, Kobayashi K, et al. Gefitinib or chemotherapy for non-small-cell lung cancer with mutated EGFR. N Engl J Med 2010; 362: 2380-2388.

40 Mitsudomi T, Morita S, Yatabe Y, et al. Gefitinib versus cisplatin plus docetaxel in patients with non-small-cell lung cancer harbouring mutations of the epidermal growth factor receptor (WJTOG3405): an open label, randomised phase 3 trial. Lancet Oncol 2010; 11: 121-128.

41 Rosell R, Carcereny E, Gervais R, et al. Erlotinib versus standard chemotherapy as first-line treatment for European patients with advanced EGFR mutation-positive non-small-cell lung cancer (EURTAC): a multicentre, open-label, randomised phase 3 trial. Lancet Oncol 2012; 13: 239-246. 
42 Zhou C, Wu YL, Cheng G, et al. Erlotinib versus chemotherapy as first-line treatment for patients with advanced EGFR mutation-positive non-small-cell lung cancer (OPTIMAL, CTONG-0802): a multicentre, open-label, randomised, phase 3 study. Lancet Oncol 2011; 12: 735-742.

43 Sequist LV, Yang JC, Yamamoto N, et al. Phase III study of afatinib or cisplatin plus pemetrexed in patients with metastatic lung adenocarcinoma with EGFR mutations. J Clin Oncol 2013; 31: 3327-3334.

44 Broglio KR, Berry DA. Detecting an overall survival benefit that is derived from progression-free survival. $J$ Natl Cancer Inst 2009; 101: 1642-1649.

45 Ou SH, Janne PA, Bartlett CH, et al. Clinical benefit of continuing ALK inhibition with crizotinib beyond initial disease progression in patients with advanced ALK-positive NSCLC. Ann Oncol 2014; 25: 415-422.

46 Von Hoff DD, Stephenson JJ, Rosen P, et al. Pilot study using molecular profiling of patients' tumors to find potential targets and select treatments for their refractory cancers. J Clin Oncol 2010; 28: 4877-4883.

47 Langer NH, Christensen TN, Langer SW, et al. PET/CT in therapy evaluation of patients with lung cancer. Expert Rev Anticancer Ther 2014; 14: 595-620.

48 Kanazu M, Maruyama K, Ando M, et al. Early pharmacodynamic assessment using ${ }^{18} \mathrm{~F}$-fluorodeoxyglucose positron-emission tomography on molecular targeted therapy and cytotoxic chemotherapy for clinical outcome prediction. Clin Lung Cancer 2014; 15: 182-187.

49 Hachemi M, Couturier O, Vervueren L, et al. $\left[{ }^{18} \mathrm{~F}\right] \mathrm{FDG}$ positron emission tomography within two weeks of starting erlotinib therapy can predict response in non-small cell lung cancer patients. PLoS One 2014; 9: e87629.

50 Yoon SH, Goo JM, Lee SM, et al. Positron emission tomography/magnetic resonance imaging evaluation of lung cancer. J Thorac Imaging 2014; 29: 4-16.

51 Scheffler M, Zander T, Nogova L, et al. Prognostic impact of $\left[{ }^{18} \mathrm{~F}\right]$ fluorothymidine and $\left[{ }^{18} \mathrm{~F}\right]$ fluoro-D-glucose baseline uptakes in patients with lung cancer treated first-line with erlotinib. PLoS One 2013; 8: e53081.

52 Kahraman D, Holstein A, Scheffler M, et al. Tumor lesion glycolysis and tumor lesion proliferation for response prediction and prognostic differentiation in patients with advanced non-small cell lung cancer treated with erlotinib. Clin Nucl Med 2012; 37: 1058-1064.

53 Scheffler M, Kobe C, Zander T, et al. Monitoring reversible and irreversible EGFR inhibition with erlotinib and afatinib in a patient with EGFR-mutated non-small cell lung cancer (NSCLC) using sequential $\left[{ }^{18} \mathrm{~F}\right]$ fluorothymidine (FLT-)PET. Lung Cancer 2012; 77: 617-620.

54 Trigonis I, Koh PK, Taylor B, et al. Early reduction in tumour $\left[{ }^{18} \mathrm{~F}\right]$ fluorothymidine (FLT) uptake in patients with non-small cell lung cancer (NSCLC) treated with radiotherapy alone. Eur J Nucl Med Mol Imaging 2014; 41: 682-693.

55 Everitt SJ, Ball DL, Hicks RJ, et al. Differential ${ }^{18} \mathrm{~F}-\mathrm{FDG}$ and ${ }^{18} \mathrm{~F}-\mathrm{FLT}$ uptake on serial PET/CT imaging before and during definitive chemoradiation for non-small cell lung cancer. J Nucl Med 2014 [In press DOI: 10.2967/ jnumed.113.131631].

56 Tsuchida T, Morikawa M, Demura Y, et al. Imaging the early response to chemotherapy in advanced lung cancer with diffusion-weighted magnetic resonance imaging compared to fluorine-18 fluorodeoxyglucose positron emission tomography and computed tomography. J Magn Reson Imaging 2013; 38: 80-88.

57 van der Veldt AA, Meijerink MR, van den Eertwegh AJ, et al. Targeted therapies in renal cell cancer: recent developments in imaging. Target Oncol 2010; 5: 95-112.

58 Wang JW, Zheng W, Liu JB, et al. Assessment of early tumor response to cytotoxic chemotherapy with dynamic contrast-enhanced ultrasound in human breast cancer xenografts. PLoS One 2013; 8: e58274.

59 Hwang M, Niermann KJ, Lyshchik A, et al. Sonographic assessment of tumor response: from in vivo models to clinical applications. Ultrasound Q 2009; 25: 175-183.

60 Lassau N, Chebil M, Chami L, et al. Dynamic contrast-enhanced ultrasonography (DCE-US): a new tool for the early evaluation of antiangiogenic treatment. Target Oncol 2010; 5: 53-58.

61 Garcia-Figueiras R, Goh VJ, Padhani AR, et al. CT perfusion in oncologic imaging: a useful tool? AJR Am J Roentgenol 2013; 200: 8-19.

62 van Elmpt W, Das M, Huellner M, et al. Characterization of tumor heterogeneity using dynamic contrast enhanced CT and FDG-PET in non-small cell lung cancer. Radiother Oncol 2013; 109: 65-70.

63 Robert C, Soria JC, Eggermont AMM. Drug of the year: programmed death-1 receptor/programmed death-1 ligand-1 receptor monoclonal antibodies. Eur J Cancer 2013; 49: 2968-2971.

64 Champiat S, Ileana E, Giaccone G, et al. Incorporating immune-checkpoint inhibitors into systemic therapy of NSCLC. I Thorac Oncol 2014; 9: 144-153.

65 Topalian SL, Hodi FS, Brahmer JR, et al. Safety, activity, and immune correlates of anti-PD-1 antibody in cancer. N Engl J Med 2012; 366: 2443-2454.

66 Brahmer JR, Tykodi SS, Chow LQ, et al. Safety and activity of anti-PD-L1 antibody in patients with advanced cancer. N Engl J Med 2012; 366: 2455-2465.

67 Spigel DR, Gettinger SN, Horn L, et al. Clinical activity, safety, and biomarkers of MPDL3280A, an engineered PDL1 antibody in patients with locally advanced or metastatic non-small cell lung cancer (NSCLC). J Clin Oncol 2013; 31: 8008 .

68 Herbst RS, Gordon MS, Fine GD, et al. A study of MPDL3208A, an engineered PD-L1 antibody in patients with locally advanced or metastatic tumors. J Clin Oncol 2013; 31: 3000.

69 Ribas A, Chmielowski B, Glaspy JA. Do we need a different set of response assessment criteria for tumor immunotherapy? Clin Cancer Res 2009; 15: 7116-7118.

70 Hoos A, Eggermont AM, Janetzki S, et al. Improved endpoints for cancer immunotherapy trials. J Natl Cancer Inst 2010; 102: 1388-1397.

71 Wolchok JD, Hoos A, O'Day S, et al. Guidelines for the evaluation of immune therapy activity in solid tumors: immune-related response criteria. Clin Cancer Res 2009; 15: 7412-7420.

72 Gandara DR, Li T, Lara PN, et al. Acquired resistance to targeted therapies against oncogene-driven non-small cell lung cancer: approach to subtyping progressive disease and clinical implications. Clin Lung Cancer 2014; 15: 1-6.

73 Kris MG, Johnson B, Kwiatkowski DJ, et al. Identification of driver mutations in tumor specimens from 1,000 patients with lung adenocarcinoma: the NCI's Lung Cancer Mutation Consortium (LCMC). J Clin Oncol 2011; 29: CRA7506.

74 MacConaill LE, Garraway LA. Clinical implications of the cancer genome. J Clin Oncol 2010; 28: 5219-5228.

75 MacConaill LE. Existing and emerging technologies for tumor genomic profiling. J Clin Oncol 2013; 31: 1815-182 4. 